\title{
GENERIC CONTINUITY OF MINIMAL SET-VALUED MAPPINGS
}

\author{
W. B. MOORS and J. R. GILES
}

(Received 20 August 1996)

Communicated by E. N. Dancer

\begin{abstract}
We study classes of Banach spaces where every set-valued mapping from a complete metric space into subsets of the Banach space which satisfies certain minimal properties, is single-valued and norm upper semi-continuous at the points of a dense $G_{\delta}$ subset of its domain. Characterisations of these classes are developed and permanence properties are established. Sufficiency conditions for membership of these classes are defined in terms of fragmentability and $\sigma$-fragmentability of the weak topology. A characterisation of non membership is used to show that $\ell_{\infty}(\mathbb{N})$ is not a member of our classes of generic continuity spaces.
\end{abstract}

1991 Mathematics subject classification (Amer. Math. Soc.): primary 46B22; secondary 46B20, 58C20.

\section{Introduction}

Considerable recent research has developed about the problem of determining classes of Banach spaces where the continuous convex functions have desirable differentiability properties. The significance of this research was established when it was seen that it is closely allied to the problem of determining classes of Banach spaces which possess the Radon-Nikodým Property. It was shown [2] that a Banach space has the RadonNikodým Property if and only if every continuous weak * lower semi-continuous convex function on an open convex subset of the dual is Fréchet differentiable on a dense $G_{\delta}$ subset of its domain. Recently it has been shown [8] that there is a class of Banach spaces larger than the class of spaces with the Radon-Nikodým Property where every continuous convex function on an open convex subset of the dual possessing

The authors wish to express their thanks to CECM, Simon Fraser University for hospitality while this research was being undertaken.

The second author wishes to express his thanks to the University of Washington where this research was initiated while he was a visiting scholar.

(C) 1997 Australian Mathematical Society 0263-6115/97\$A2.00+0.00 
a weak * continuous subgradient at the points of a residual subset of its domain is Fréchet differentiable on a dense $G_{\delta}$ subset of its domain.

The differentiability of a continuous convex function is associated with singlevaluedness and continuity properties of the subdifferential mapping of the function which is a set-valued mapping from the domain into the set of subgradients of the function. This association suggests a generalisation into the study of single-valuedness and continuity properties of certain set-valued mappings which we call minimal mappings. In this regard this paper can be considered as a sequel to the paper [8] developing this generalisation and answering some of the questions raised by that paper.

Fragmentability conditions are an important tool in determining differentiability properties. In particular, a Banach space has the Radon-Nikodým Property if and only if every non-empty bounded subset has slices of arbitrarily small diameter. In the paper [8] it was shown that a Banach space belongs to our class if it satisfies an appropriately weaker fragmentability condition by a metric whose topology on bounded sets is stronger than the weak topology. Recently, in papers $[10,11]$ there has been considerable development of the more general concept of $\sigma$-fragmentability and we show that a Banach space belongs to our class if it satisfies similar $\sigma$-fragmentability conditions.

Variants of the Banach-Mazur game technique are now more widely used, especially since the spectacular success achieved in the paper [17]. The technique was used in paper [8] to find a space which does not belong to our class and here we use the technique in association with Namioka's work on $\sigma$-fragmentability to show that $\ell_{\infty}(\mathbb{N})$ does not belong to our class.

\section{Minimal and hyperplane minimal mappings}

An important class of set-valued mappings consists of the upper semi-continuous mappings. A set-valued mapping $\phi$ from a topological space $A$ into non-empty subsets of a topological space $X$ is upper semi-continuous at $t \in A$ if for every open set $W$ such that $\phi(t) \subseteq W$ there exists an open neighbourhood $U$ of $t$ such that $\phi(U) \subseteq W$. Such mappings with compact images are called uscos and when $X$ is a linear topological space and the mappings have convex compact images they are called cuscos.

Within these classes of mappings special attention is given to the minimal mappings, which are those whose graph does not contain the graph of any other from the class with the same domain. The following characterisation of minimality for uscos and cuscos is well known, [5, p. 252]. A usco (cusco) $\phi$ from a topological space $A$ into subsets of a Hausdorff space (separated locally convex space) is minimal if and only if, for every open set (open half space) $W$ and open set $U$ in $A$ such that $\phi(U) \cap W \neq \emptyset$ there exists a non-empty open set $V \subseteq U$ such that $\phi(V) \subseteq W$. 
Often when considering questions such as the generic single-valuedness and continuity of such mappings it is the minimality condition rather than the upper semicontinuity which plays the significant role. This suggests that we isolate the minimality condition and consider such questions for the class of what we will call minimal mappings.

We say that a set-valued mapping $\phi$ from a topological space $A$ into non-empty subsets of a topological space $X$ is minimal if for any open set $W$ in $X$ and open set $U$ in $A$ such that $\phi(U) \cap W \neq \emptyset$ there exists a non-empty open set $V \subseteq U$ such that $\phi(V) \subseteq W$. We say that a set-valued mapping $\phi$ from a topological space $A$ into non-empty subsets of a linear topological space $X$ is hyperplane minimal if for any open half-space $W$ and open set $U$ in $A$ such that $\phi(U) \cap W \neq \emptyset$ there exists a non-empty open set $V \subseteq U$ such that $\phi(V) \subseteq W$. It is easily verified that when $X$ is a topological space (locally convex space) $\phi$ is minimal (hyperplane minimal) if and only if for every open set $U$ in $A$ and closed (closed and convex) set $K$ in $X$ such that $\phi(U) \nsubseteq K$ there exists a non-empty open set $V \subseteq U$ such that $\phi(V) \cap K=\emptyset$.

We observe that these definitions lose the initial connotation of minimal because any set-valued mapping from a topological space $A$ into non-empty subsets of a topological space (linear topological space) $X$ whose graph is contained in the graph of a minimal (hyperplane minimal) mapping on $A$ is also minimal (hyperplane minimal). But also for a minimal (hyperplane minimal) mapping from a topological space $A$ into subsets of a topological space (linear topological space) $X$, the restriction to any non-empty open subset of $A$ or to any dense subset of any open subset of $A$ is also minimal (hyperplane minimal). In particular, any selection from a dense subset of an open subset of $A$ is still minimal (hyperplane minimal) and when the range space is a regular Hausdorff space (separated locally convex space) we can often determine continuity properties of a minimal (hyperplane minimal) mapping from continuity properties of such a selection.

THEOREM 1.1. Consider a minimal (hyperplane minimal) mapping $\phi$ from a topological space $A$ into subsets of a topological space (locally convex space) $X$, and a selection $\sigma$ on $A$ and a dense subset $D$ of $A$.

(i) For any open set $U$ in $A, \phi(U) \subseteq \overline{\sigma(U \cap D)}, \quad(\phi(U) \subseteq \overline{\operatorname{co}} \sigma(U \cap D))$.

(ii) If $X$ is a regular Hausdorff space (separated locally convex space) and $\left.\sigma\right|_{D}$ is continuous at $t_{0} \in D$ then $\phi$ is single-valued and upper semi-continuous at $t_{0}$.

Proof. (i) Suppose $\phi(U) \nsubseteq \overline{\sigma(U \cap D)},(\phi(U) \nsubseteq \overline{c o} \sigma(U \cap D))$. Then since $\phi$ is minimal (hyperplane minimal) there exists a non-empty open set $V \subseteq U$ such that

$$
\phi(V) \cap \overline{\sigma(U \cap D)}=\emptyset, \quad(\phi(V) \cap \overline{\operatorname{co}} \sigma(U \cap D)=\emptyset) .
$$

But this contradicts the density of $D$. 
(ii) As $X$ is a regular Hausdorff space, $\sigma\left(t_{0}\right)$ has a base of closed (closed and convex) neighbourhoods whose intersection is $\sigma\left(t_{0}\right)$. Given one such neighbourhood $N_{0}$ of $\sigma\left(t_{0}\right)$ there exists an open neighbourhood $U$ of $t_{0}$ such that $\sigma(U \cap D) \subseteq N_{0}$. Then by (i),

$$
\phi(U) \subseteq \overline{\sigma(U \cap D)} \subseteq N_{0}, \quad\left(\phi(U) \subseteq \overline{\operatorname{co}} \sigma(U \cap D) \subseteq N_{0}\right)
$$

So we conclude that $\phi$ is single-valued and upper semi-continuous at $t_{0}$.

It is convenient to have on hand elementary algebraic properties of minimal and hyperplane minimal mappings which we will use in the subsequent development of our theory.

THEOREM 1.2. Consider a minimal (hyperplane minimal) mapping $\phi$ from a topological space $A$ into subsets of a linear topological space $X$.

(i) Given a continuous (continuous linear) mapping $T$ from $X$ into a linear topological space $Y$, then $T \circ \phi$ is a minimal (hyperplane minimal) mapping from $A$ into subsets of $Y$.

(ii) Given a continuous real valued function $g$ on $A$, then $g . \phi$ is a minimal mapping from $A$ (hyperplane minimal mapping from $A \backslash g^{-1}(0)$ ) into subsets of $X$.

(iii) Given a continuous mapping $T$ from $A$ into $X$, then $T+\phi$ is a minimal (hyperplane minimal) mapping from $A$ into subsets of $X$.

Proof. (i) Consider an open set (open half space) $W$ in $Y$ and an open set $U$ in $A$ such that $(T \circ \phi)(U) \cap W \neq \emptyset$. Since $T$ is continuous on $X, T^{-1}(W)$ is an open set (open half space) in $X$. Since $\phi$ is minimal (hyperplane minimal) and $\phi(U) \cap T^{-1}(W) \neq \emptyset$ there exists a non-empty open set $V \subseteq U$ such that $\phi(V) \subseteq \phi(U) \cap T^{-1}(W)$. Then $T \circ \phi(V) \subseteq W$.

(ii) In the case when $\phi$ is a minimal mapping, $g . \phi$ is the composite of the continuous mapping $T$ from $\mathbb{R} \times X$ into $X$ defined by $T(t, x)=t . x$ with the minimal mapping $t \mapsto(g(t), \phi(t))$ from $A$ into $\mathbb{R} \times X$ and so by (i) $g . \phi$ is minimal.

In the case when $\phi$ is a hyperplane minimal mapping, consider an open set $U$ in $A \backslash g^{-1}(0)$ and an open half-space $W$ such that $g \cdot \phi(U) \cap W \neq \emptyset$. Now there exists an $f \in X^{*}$ and a real $\alpha$ such that $W \equiv\{x \in X: f(x)>\alpha\}$. Consider $t_{0} \in U$ such that $f\left(g\left(t_{0}\right) \cdot x_{0}\right)>\alpha$ for some $x_{0} \in \phi\left(t_{0}\right)$. Then there exists an $\epsilon>0$ such that $g\left(t_{0}\right) f\left(x_{0}\right)>\alpha+\epsilon$. Since $g$ is continuous at $t_{0}$ there exists a neighbourhood $U^{\prime}$ of $t_{0}, U^{\prime} \subseteq U$ such that $g(t) f\left(x_{0}\right)>\alpha+\epsilon$ for all $t \in U^{\prime}$. Consider the case when $g\left(t_{0}\right)>0$. Then we may choose $U^{\prime}$ such that $g(t)>0$ for all $t \in U^{\prime}$. Then

$$
f\left(x_{0}\right)>M \equiv \sup \left\{\alpha / g(t): t \in U^{\prime}\right\}
$$


Since $\phi$ is hyperplane minimal there exists a non-empty open set $V \subseteq U^{\prime}$ such that $f(\phi(t))>M$ for all $t \in V$. Therefore $g(t) f(\phi(t))>\alpha$ for all $t \in V$. Consider the case when $g\left(t_{0}\right)<0$. Then we may choose $U^{\prime}$ such that $g(t)<0$ for all $t \in U^{\prime}$. Then

$$
f\left(x_{0}\right)<m \equiv \inf \left\{\alpha / g(t): t \in U^{\prime}\right\} .
$$

Again, since $\phi$ is hyperplane minimal there exists a non-empty open set $V \subseteq U^{\prime}$ such that $f(\phi(t))<m$ for all $t \in V$. Therefore $g(t) f(\phi(t))>\alpha$ for all $t \in V$. In both cases, $g . \phi(V) \subseteq W$ and we conclude that $g . \phi$ is hyperplane minimal on $A \backslash g^{-1}(0)$.

(iii) The mapping $\Phi$ from $A$ into $X \times X$ defined by $\Phi(t)=(T(t), \phi(t))$ is also minimal (hyperplane minimal). Since $T+\phi$ is the composite with $\Phi$ of the continuous linear mapping from $X \times X$ into $X$ defined by $(x, y) \mapsto x+y$, we deduce from (i) that $T+\phi$ is minimal (hyperplane minimal).

We should note that in Theorem 1.2 (ii) the mapping $g . \phi$ is not necessarily hyperplane minimal on any domain which includes the set $g^{-1}(0)$. For example, consider the hyperplane minimal mapping $\phi$ on $\mathbb{R}$ defined by

$$
\phi(t)= \begin{cases}1 / t & t \neq 0 \\ 0 & t=0,\end{cases}
$$

and the continuous mapping $g$ on $\mathbb{R}$ defined by $g(t)=t$. Then

$$
g . \phi= \begin{cases}1 & t \neq 0 \\ 0 & t=0,\end{cases}
$$

which is not hyperplane minimal.

Our principal interest is in norm continuity properties of weakly minimal (hyperplane minimal) mappings from a complete metric space into subsets of a Banach space. We say that a Banach space $X$ is a general generic continuity space (GGC space) if every weakly minimal mapping $\phi$ from a complete metric space $A$ into subsets of $X$ is single-valued and norm upper semi-continuous at the points of a dense $G_{\delta}$ subset of $A$. We say that a Banach space is a generic continuity space (GC space) if every hyperplane minimal mapping $\phi$ from a complete metric space $A$ into subsets of $X$ is single-valued and norm upper semi-continuous at the points of a dense $G_{\delta}$ subset of A.

Since very weakly minimal mapping $\phi$ from a complete metric space $A$ into subsets of a Banach space $X$ is also hyperplane minimal, it is clear that the class of GGC spaces contains the class of GC spaces. However, the subdifferential mapping of the modulus function on $\mathbb{R}$ is hyperplane minimal but not minimal, and so the further relation between the classes is open to investigation. 
In this paper we study the class of GGC spaces and the class of GC spaces. In paper [8] the class of GC spaces was introduced but geometrical conditions of a particular type implying membership of the class had been given in papers [13], [5, 6, 7] and [14]. It was shown that all separable, all weakly compactly generated spaces and those with the Radon-Nikodým property belong to this class as do those which can be equivalently renormed to be weakly locally uniformly rotund or whose dual belongs to Stegall's class $\mathscr{S}$. In paper [8] it was shown that $\ell_{\infty}(\Gamma)$ where $\Gamma$ is uncountable is not a GC space. Subsequently, in private communication, Namioka showed that $\ell_{\infty}(\mathbb{N})$ is not a GGC space, and it was at his suggestion that we began to examine the class of GGC spaces.

We begin with characterisations of the class of GGC and GC spaces which link them to earlier work and which make them more amenable to study. We then examine hereditary and three space properties for the classes. In paper [8], fragmentability conditions were given sufficient for a Banach space to be a GC space. To show that $\ell_{\infty}(\mathbb{N})$ is not a GGC space, Namioka used recent work on $\sigma$-fragmentability. This suggested that we study $\sigma$-fragmentability conditions sufficient for a Banach space to be a GGC space. Finally we give a characterisation for non-membership of our classes in terms of a Banach-Mazur game and use this to present Namioka's example.

We are indebted to Professor Isaac Namioka for his interest and discussions on the subject of this paper and for providing the example which motivated our research.

\section{Characterisations of the classes of GGC and GC spaces}

The study of GC spaces began as an investigation of the differentiability properties of continuous convex functions on open convex subsets of the dual of a Banach space. The subdifferential mapping of a continuous convex function on an open convex subset of a Banach space is a minimal weak * cusco into subsets of the dual of the space, $[16$, p. 105], and it is well known that the convex function is Fréchet differentiable at a point of its domain if and only if its subdifferential mapping is single-valued and norm upper semi-continuous at the point, $[16$, p. 19]. So naturally interest focused on single-valued and norm upper semi-continuous properties of certain minimal weak * cuscos from a complete metric space into subsets of the second dual. However, it can be seen that manipulation of the theory is made considerably simpler by studying minimal mappings into subsets of the original space. The basis of this connection is given in Theorems 2.5 and 2.8 .

We begin by developing particularly useful properties for hyperplane minimal mappings into subsets of a Banach space similar to those which hold for minimal weak * cuscos into the dual of a Banach space, [13, p. 471]. Given a normed linear space $X$ we will denote by $\tau$ the weak topology on $X$, or when $X$ is a dual space the 
weak or weak * topology on $X$.

LEMMA 2.1. Given a $\tau$-hyperplane minimal mapping $\phi$ from a Baire space $A$ into subsets of a Banach space $X$ there exists a dense $G_{\delta}$ subset $D$ of $A$ where for each $t \in D$, the real valued function defined on $A$ by $\rho(t)=\inf \{\|x\|: x \in \phi(t)\}$ is continuous and $\phi(t)$ lies in the face of a sphere of $X$ of radius $\rho(t)$.

PRoOF. Given $\alpha \in \mathbb{R}$ consider the set $A_{\alpha} \equiv\{t \in A: \rho(t)>\alpha\}$. Since the norm on $X$ is $\tau$-lower semi-continuous the set $W \equiv\{x \in X:\|x\|>\alpha\}$ is $\tau$-open in $X$. Since $\phi$ is $\tau$-hyperplane minimal on $A$, for $t_{0} \in A_{\alpha}$ and neighbourhood $U$ of $t_{0}$ there exists a non-empty open set $V \subseteq U$ such that $\phi(V) \subseteq W$, so $\rho(t)>\alpha$ for all $t \in V$. Since $A$ is a Baire space, we deduce that $\rho$ is continuous at the points of a dense $G_{\delta}$ subset $D$ of $A$, [4, Theorem 1.5].

Suppose that for some $t_{0} \in D$ there exists an $x_{0} \in \phi\left(t_{0}\right)$ such that $\left\|x_{0}\right\|>\rho\left(t_{0}\right)$. Then given $\left\|x_{0}\right\|>r>\rho\left(t_{0}\right)$, since $\rho$ is continuous at $t_{0}$ there exists an open neighbourhood $U$ of $t_{0}$ such that $\rho(t)<r$ for all $t \in U$. But $\phi(U) \nsubseteq B[0 ; r]$ and since $\phi$ is $\tau$-hyperplane minimal and $B[0 ; r]$ is $\tau$-closed there exists a non-empty open set $V \subseteq U$ such that $\phi(V) \cap B[0 ; r]=\emptyset$. But then $\rho(t) \geq r$ for all $t \in V$ which is a contradiction. So we conclude that $\|x\|=\rho(t)$ for all $t \in D$.

This result important in itself, has a significant consequence.

A set-valued mapping $\phi$ from a topological space $A$ into subsets of a Banach space $X$ is said to be locally bounded on a subset $D$ of $A$ if for each $t \in D$ there exists a neighbourhood $U$ of $t$ and a $K>0$ such that $\|x\| \leq K$ for all $x \in \phi(U)$.

COROLLARY 2.2. A $\tau$-hyperplane minimal mapping $\phi$ from a Baire space $A$ into subsets of a Banach space $X$ is locally bounded at the points of a dense open subset of $A$.

PROOF. We show that $\phi$ is locally bounded at the points of the subset $D$ of $A$ where $\rho$ is continuous. Consider $t_{0} \in D$ and $\epsilon>0$. Since $\rho$ is continuous at $t_{0}$ there exists a neighbourhood $U$ of $t_{0}$ such that

$$
\phi(t) \cap B\left[0 ; \rho\left(t_{0}\right)+\epsilon\right] \neq \emptyset \quad \text { for each } t \in U .
$$

Suppose that $\phi(U) \nsubseteq B\left[0 ; \rho\left(t_{0}\right)+\epsilon\right]$. Since $\phi$ is $\tau$-hyperplane minimal and $B\left[0 ; \rho\left(t_{0}\right)+\epsilon\right]$ is $\tau$-closed there exists a non-empty open set $V \subseteq U$ such that $\phi(V) \cap B\left[0 ; \rho\left(t_{0}\right)+\epsilon\right]=\emptyset$, which is a contradiction.

The next lemma relates locally bounded weakly minimal and hyperplane minimal mappings into subsets of a Banach space to minimal weak * uscos and minimal weak * cuscos into subsets of the second dual of a Banach space. 
LEMMA 2.3. Consider a Banach space $X$ with second dual $X^{* *}$.

(i) Given $\phi$ a locally bounded weakly minimal (hyperplane minimal) mapping from a topological space $A$ into subsets of $X$, the set-valued mapping $\Phi$ from $A$ into subsets of $X^{* *}$ defined by

$$
\begin{gathered}
\Phi(t)=\bigcap\left\{\overline{\phi(U)}^{w^{*}}: U \text { is a neighbourhood of } t\right\} \\
\left(\Phi(t)=\bigcap\left\{\overline{\operatorname{co}}^{w^{*}} \phi(U): U \text { is a neighbourhood of } t\right\}\right)
\end{gathered}
$$

is a minimal weak * usco (minimal weak * cusco), [1, p. 472].

(ii) Given $\Phi$ a minimal weak * usco (minimal weak * cusco) from a topological space $A$ into subsets of $X^{* *}$ where the set $D \equiv\{t \in A: \Phi(t) \cap \widehat{X} \neq \emptyset\}$ is dense in $A$, the set-valued mapping $\phi$ from $D$ into subsets of $X$ defined by $\phi(t)=\Phi(t) \cap \widehat{X}$ is weakly minimal (hyperplane minimal) on D.

The continuity property we are interested in is deduced from Theorem 1.1 and Namioka's result $[15$, p. 525].

LEMMA 2.4. Given a weakly minimal (hyperplane minimal) mapping $\phi$ from a complete metric space A into subsets of a Banach space $X$, if there exists a dense $G_{\delta}$ subset $D$ of $A$ and a selection $\sigma$ where $\left.\sigma\right|_{D}$ is weakly continuous on $D$ then $\phi$ is single-valued and norm upper semi-continuous at the points of a dense $G_{\delta}$ subset of D.

Corollary 2.2 and Lemmas 2.3 and 2.4 enable us to establish our first characterisation of the classes of GGC and GC spaces and to show that our definition is consistent with that given for GC spaces in [8, p. 423].

THEOREM 2.5. A Banach space $X$ is GGC space (GC space) if and only if every minimal weak * usco (minimal weak * cusco) $\Phi$ from a complete metric space $A$ into subsets of $X^{* *}$ where the set $\{t \in A: \Phi(t) \cap \widehat{X} \neq \emptyset\}$ is residual in $A$ is single-valued and norm upper semi-continuous at the points of a dense $G_{\delta}$ subset of $A$.

We will find it useful to have a characterisation for these spaces in terms of minimal mappings into the unit sphere of the space. We do this by using Lemma 2.1.

Given a weakly minimal (hyperplane minimal) mapping $\phi$ from a Baire space $A$ into subsets of a Banach space $X$, using the dense $G_{\delta}$ subset $D$ of $A$ given in Lemma 2.1, when $D \backslash \rho^{-1}(0) \neq \emptyset$ we define the mapping $\hat{\phi}$ from $D \backslash \rho^{-1}(0)$ into subsets of $S(X)$ by $\hat{\phi}(t)=\phi(t) / \rho(t)$. The mapping $\hat{\phi}$ on $D \backslash \rho^{-1}(0)$ inherits minimality properties from the mapping $\phi$ which generates it. 
LEMMA 2.6. Given a weakly minimal (hyperplane minimal) mapping $\phi$ from a Baire space $A$ into subsets of a Banach space $X$, when $D \backslash \rho^{-1}(0) \neq \emptyset$, the associated mapping $\hat{\phi}$ from $D \backslash \rho^{-1}(0)$ into subsets of $S(X)$ is weakly minimal (hyperplane minimal).

PROOF. Now $\phi$ restricted to the dense subset $D$ is still weakly minimal (hyperplane minimal). Since $\rho^{-1}(0)$ is a closed subset of $D$, then $D \backslash \rho^{-1}(0)$ is an open subset of $D$ and we see that $\phi$ restricted to $D \backslash \rho^{-1}(0)$ is still weakly minimal (hyperplane minimal). Clearly, $\hat{\phi}$ is the product of the continuous real-valued function $1 / \rho$ on $D \backslash \rho^{-1}(0)$ with $\phi$ restricted to $D \backslash \rho^{-1}(0)$ so by Theorem 1.2 (ii), $\hat{\phi}$ is a weakly minimal (hyperplane minimal) mapping on $D \backslash \rho^{-1}(0)$.

LEMMA 2.7. Consider a hyperplane minimal mapping $\phi$ from a Baire space $A$ into subsets of a Banach space $X$ where $D \backslash \rho^{-1}(0) \neq \emptyset$. If the associated mapping $\hat{\phi}$ from $D \backslash \rho^{-1}(0)$ into subsets of $S(X)$ is single-valued and norm upper semi-continuous at the points of a dense $G_{\delta}$ subset $D^{\prime}$ of $D \backslash \rho^{-1}(0)$ then $\phi$ is also single-valued and norm upper semi-continuous at the points of $D^{\prime} \cup\left(\rho^{-1}(0) \cap D\right)$ which is a residual subset of $A$.

PROOF. Since $\rho$ is continuous at the points of $D$ and $\hat{\phi}$ is single-valued and norm upper semi-continuous at the points of $D^{\prime}$, then $\left.\phi\right|_{D \backslash \rho^{-1}(0)}$ is single-valued and norm upper semi-continuous at the points of $D^{\prime}$. Since $D \backslash \rho^{-1}(0)$ is a relatively open subset of $D,\left.\phi\right|_{D}$ is single-valued and norm upper semi-continuous at the points of $D^{\prime}$.

For $t_{0} \in \rho^{-1}(0) \cap D$, since $\rho$ is continuous at $t_{0}$ there exists a neighbourhood $U$ of $t_{0}$ such that $\rho(t)<\epsilon$ for all $t \in U$. Then $\phi(t) \subseteq B(0 ; \epsilon)$ for all $t \in U \cap D$, so $\left.\phi\right|_{D}$ is single-valued and norm upper semi-continuous at the points of $D^{\prime} \cup\left(\rho^{-1}(0) \cap D\right)$. But again since $D$ is dense in $A$ and $\phi$ is hyperplane minimal on $A$, by Theorem 1.1, $\phi$ is single-valued and norm upper semi-continuous at the points of $D^{\prime} \cup\left(\rho^{-1}(0) \cap D\right)$.

We should note that if $D \subseteq \rho^{-1}(0)$ then $\phi$ is single-valued and maps to 0 on $D$. Then $\left.\phi\right|_{D}$ is norm continuous on $D$ and by Theorem 1.1, $\phi$ is single-valued and norm upper semi-continuous at the points of $D$.

Consider the case when $A$ is a complete metric space. Since $D$ is a $G_{\delta}$ subset of $A$ it is completely metrisable and since $D \backslash \rho^{-1}(0)$ is an open subset of $D$ it is also completely metrisable. So we have the following characterisation for GGC and GC spaces.

THEOREM 2.8. A Banach space $X$ is a GGC space (GC space) if and only if every weakly minimal (hyperplane minimal) mapping $\phi$ from a complete metric space $A$ into subsets of $S(X)$ is single-valued and norm upper semi-continuous at the points of a dense $G_{\delta}$ subset of $A$. 
We will use the characterisations Theorem 2.5 in Section 3 and Theorem 2.8 in Section 4. It will often be convenient to work with selections as in Theorem 3.7. It will also be useful to consider mappings into subsets of the closed unit ball as we do in Theorem 3.9 and Section 5. For convenience we list these easily established subsidiary characterisations.

THEOREM 2.9. For a Banach space $X$, the following are equivalent:

(i) $X$ is a GGC space (GC space).

(ii) Every minimal weak * usco (minimal weak * cusco) $\Phi$ from a complete metric space $A$ into subsets of $B\left(X^{* *}\right)$ (or into subsets of $S\left(X^{* *}\right)$ ) where the set $\{t \in A: \Phi(t) \cap \widehat{X} \neq \emptyset\}$ is residual in $A$, is single-valued and norm upper semicontinuous at the points of a dense $G_{\delta}$ subset of $A$.

(iii) Every weakly minimal (hyperplane minimal) mapping $\phi$ from a complete metric space $A$ into subsets of $B(X)$ (or into subsets of $S(X)$ ) is single-valued and norm upper semi-continuous at the points of a dense $G_{\delta}$ subset of $A$.

(iv) Every single-valued weakly minimal (hyperplane minimal) mapping $\sigma$ from a complete metric space $A$ into $X($ or into $B(X)$, or into $S(X)$ ) is weakly continuous at the points of a dense $G_{\delta}$ subset of $A$.

\section{Properties of the classes of GGC and GC spaces}

In Section 1 we mentioned that the class of GGC spaces contains the class of GC spaces. We use the characterisation in Theorem 2.5 to show that when we restrict ourselves to Banach spaces satisfying a particular geometrical property, the two classes coincide.

The following relation between minimal weak * cuscos and weak * uscos gives us some insight.

LEMMA 3.1. In a Banach space $X$ which can be equivalently renormed to have every point of $S(\widehat{X})$ an extreme point of $B\left(X^{* *}\right)$, for any minimal weak * cusco $\Phi$ from a Baire space $A$ into subsets of $X^{* *}$ and minimal weak * usco $\Psi$ from $A$ whose graph is contained in that of $\Phi$, the set

$$
\{t \in A: \Phi(t) \cap \widehat{X} \neq \emptyset\} \backslash\{t \in A: \Psi(t) \cap \widehat{X} \neq \emptyset\}
$$

is first category in A.

PROOF. Consider $X$ so renormed. Given $t \in A, \Phi(t)=\overline{c o}^{w^{*}} \Psi(t)$ [1, p. 462]. Since $\Phi(t)$ is weak * compact, ext $\Phi(t) \subseteq \Psi(t)$. Write $E \equiv\{t \in A: \Phi(t) \cap \widehat{X} \neq \emptyset\}$. Now the set $T \equiv\left\{t \in A: \Phi(t)\right.$ lies in the face of a sphere of $\left.X^{* *}\right\}$ is residual in $A$. So for 
$t \in T \cap E$, any point $F \in \Phi(t) \cap \widehat{X}$ is an extreme point of $\|F\| B\left(X^{* *}\right)$ so is an extreme point of $\Phi(t)$ and therefore lies in $\Psi(t)$. Hence the set $\{t \in A: \Psi(t) \cap \widehat{X} \neq \emptyset\} \supseteq$ $T \cap E$. But $E \backslash T \subseteq A \backslash T$ which is first category in $A$, so $E \backslash\{t \in A: \Psi(t) \cap \widehat{X} \neq \emptyset\}$ is also first category in $A$.

Using Lemma 2.3, and noting in the above proof that for every $t \in T \cap E$, we have $\Phi(t) \cap \widehat{X}=\Psi(t) \cap \widehat{X}$ a singleton, this result has the following consequences for minimal mappings.

COROLLARY 3.2. In a Banach space $X$ which can be equivalently renormed to have every point of $S(\widehat{X})$ an extreme point of $B\left(X^{* *}\right)$, given a hyperplane minimal mapping $\phi$ from a Baire space $A$ into subsets of a Banach space $X$, there exists a dense $G_{\delta}$ subset $D$ of $A$ and a weakly minimal mapping $\psi$ from $D$ into subsets of $X$ whose graph is contained in that of $\left.\phi\right|_{D}$.

Lemma 3.1 enables us to identify a class of Banach spaces where the two classes of generic continuity spaces coincide.

THEOREM 3.3. A Banach space which can be equivalently renormed to have every point of $S(\widehat{X})$ an extreme point of $B\left(X^{* *}\right)$, is a GC space if and only if it is a GGC space.

A Banach space $X$ is said to be weakly mid-point locally uniformly rotund if for each $x \in S(X)$ and $f \in X^{*},\|f\|=1$, given $\epsilon>0$ there exists a $\delta(\epsilon, x, f)>0$ such that for all $y, z \in X,\|y\|,\|z\| \leq 1$, when $\|y+z-2 x\|<\delta$ we have $|f(y-z)|<\epsilon$. It has been shown [19] that the space being weakly mid-point locally uniformly rotund is equivalent to the space possessing the geometrical properties we require for Theorem 3.3. The original proof used the Principle of Local Reflexivity but here we give a proof using Goldstine's Theorem.

PROPOSITION 3.4. A Banach space $X$ is weakly mid-point locally uniformly rotund if and only if every point of $S(\widehat{X})$ is an extreme point of $B\left(X^{* *}\right)$.

PROOF. Suppose there exist $F_{1}, F_{2} \in X^{* *}, F_{1} \neq F_{2},\left\|F_{1}\right\|=\left\|F_{2}\right\|=1$ such that $\hat{x}=\left(F_{1}+F_{2}\right) / 2$ and $\|x\|=1$. Then there exists an $f \in X^{*},\|f\|=1$ and real $r>0$ such that $F_{1} \in W_{1} \equiv\left\{F \in X^{* *}: f(F)>f(x)+r\right\}$ and $F_{2} \in W_{2} \equiv$ $\left\{F \in X^{* *}: f(F)<f(x)-r\right\}$. Consider the convex set $W \equiv\left(W_{1} \cap B(\widehat{X})\right) / 2+$ $\left(W_{2} \cap B(\widehat{X})\right) / 2$. We will show that $\hat{x} \in \bar{W}$. Every weak neighbourhood of 0 in $X$ can be considered to be of the form $N \cap \widehat{X}$ where $N$ is a weak * neighbourhood of 0 in $X^{* *}$. Given a weak * open convex neighbourhood $N$ of 0 in $X^{* *}$, consider $\hat{x}+N \cap \widehat{X}$ a weak open neighbourhood of $\hat{x}$ in $\widehat{X}$. By Goldstine's Theorem $B(\widehat{X})$ is weak * 
dense in $B\left(X^{* *}\right)$ so there exists $\hat{y} \in\left(F_{1}+N\right) \cap W_{1}$ and $\hat{z} \in\left(F_{2}+N\right) \cap W_{2}$ where $\|y\|,\|z\| \leq 1$ and then $(\hat{y}+\hat{z}) / 2 \in\left(F_{1}+F_{2}\right) / 2+N$. So $(\hat{y}+\hat{z}) / 2 \in \hat{x}+N \cap \widehat{X}$, and we conclude that $\hat{x} \in \bar{W}$ since $W$ is convex. So given $\delta>0$ there exist $\hat{y} \in W_{1} \cap B(\widehat{X})$ and $\hat{z} \in W_{2} \cap B(\widehat{X})$ such that $\|y+z-2 x\|<\delta$. But $|f(y-z)|>2 r$ for all $\hat{y} \in W_{1}$ and $\hat{z} \in W_{2}$. Therefore $X$ is not weakly mid-point locally uniformly rotund.

Conversely, suppose that $X$ is not weakly mid-point locally uniformly rotund. Then for some $x \in S(X)$ there exists an $f \in X^{*},\|f\|=1$ and $r>0$ and sequences $\left\{y_{n}\right\},\left\{z_{n}\right\}$ in $B(X)$ such that $\left\|y_{n}+z_{n}-2 x\right\| \rightarrow 0$ as $n \rightarrow \infty$ but $\left|f\left(y_{n}-z_{n}\right)\right|>2 r$ for all $n \in \mathbb{N}$. Then $\left|f\left(y_{n}-x\right)\right|>r$ and $\left|f\left(z_{n}-x\right)\right|>r$ for sufficiently large $n \in \mathbb{N}$. Consider the weak * compact convex sets

$C_{1} \equiv\left\{F \in B\left(X^{* *}\right): F(f) \geq f(x)+r\right\} \quad$ and $\quad C_{2} \equiv\left\{F \in B\left(X^{* *}\right): F(f) \leq f(x)-r\right\}$.

Now co $\left(C_{1} \cup C_{2}\right)$ is weak * closed. We may suppose that $y_{n} \in C_{1}$ and $z_{n} \in C_{2}$ for all $n \in \mathbb{N}$. So $x \in \operatorname{co}\left(C_{1} \cup C_{2}\right)$ but $x \notin C_{1} \cup C_{2}$. So we conclude that $\hat{x}$ is not an extreme point of $B\left(X^{* *}\right)$.

So within the class of Banach spaces which can be equivalently renormed to be weakly mid-point locally uniformly rotund, the two classes of generic continuity spaces coincide.

We now show that the classes of GGC spaces and GC spaces are invariant under topological isomorphisms.

THEOREM 3.5. A Banach space $X$ is a GGC space (GC space) if it is topologically isomorphic to a closed linear subspace of a GGC space (GC space) $Y$.

PROOF. Consider a weakly minimal (hyperplane minimal) mapping $\phi$ from a complete metric space $A$ into subsets of $X$ and a topological isomorphism $T$ of $X$ into $Y$. From Theorem 1.2 (i) we see that $T \circ \phi$ is a weakly minimal (hyperplane minimal) mapping from $A$ into subsets of $Y$. But since $Y$ is a GGC space (GC space) there exists a dense $G_{\delta}$ subset $D$ of $A$ at the points of which $T \circ \phi$ is single-valued and norm upper semi-continuous. Since $T$ is a topological isomorphism, $\phi$ is single-valued and norm upper semi-continuous at the points of $D$ and we conclude that $X$ is a GGC space (GC space).

As an immediate application we have that the GGC and GC properties are hereditary.

COROLLARY 3.6. A closed linear subspace of a GGC space (GC space) is also a GGC space (GC space). 
We mentioned in Section 1 that Namioka has proved that $\ell_{\infty}(\mathbb{N})$ is not GGC space. It follows from Theorem 3.5 that any Banach space which contains a subspace topologically isomorphic to $\ell_{\infty}(\mathbb{N})$ is not a GGC space. In [8, Section 3] it was shown that $\ell_{\infty}(\Gamma)$ where $\Gamma$ is uncountable, is not a GC space. It now follows that $\ell_{\infty}(\Gamma)$ where $\Gamma$ is infinite is not a GGC space.

We now explore the GGC space and GC properties of a Banach space whose image under a continuous linear mapping is a GGC or GC space.

THEOREM 3.7. Consider a continuous linear mapping $T$ from a Banach space $X$ onto a GGC space (GC space) $Y$. If ker $T$ is a GGC space (GC space) then $X$ is a GGC space (GC space).

PROOF. By Theorem 2.9 (iv), it is sufficient to consider a single-valued weakly minimal (hyperplane minimal) mapping $\phi$ from a complete metric space $A$ into $X$. Consider the mapping $T \circ \phi$ from $A$ into $Y$. Since $T$ is continuous, by Theorem 1.2 (i), $T \circ \phi$ is weakly minimal (hyperplane minimal) on $A$ and since $Y$ is a GGC space (GC space) there exists a dense $G_{\delta}$ subset $D$ of $A$ where $T \circ \phi$ is norm continuous. Now $D$ is completely metrisable and $\left.T \circ \phi\right|_{D}$ is norm continuous on $D$. By the Bartle-Graves Theorem there exists a continuous mapping $\chi$ from $Y$ into $X$ such that $T \circ \chi$ is the identity on $Y$. Consider the mappings $S$ from $D$ into $X$ defined by $S=\left.\phi\right|_{D}-\chi \circ\left(\left.T \circ \phi\right|_{D}\right)$. Now $\phi$ is weakly minimal (hyperplane minimal) on $D$ and since $\chi \circ\left(\left.T \circ \phi\right|_{D}\right)$ is continuous on $D$ we have from Theorem 1.2 (iii) that $S$ is weakly minimal (hyperplane minimal) on $D$. Furthermore, we note that $S(D) \subseteq \operatorname{ker} T$. Since $\operatorname{ker} T$ is a GGC space (GC space) it follows that there exists a dense $G_{\delta}$ subset $D^{\prime}$ of $D$ at the points of which $S$ is norm continuous. But since $\left.\phi\right|_{D}=S+\chi \circ\left(\left.T \circ \phi\right|_{D}\right)$ then $\left.\phi\right|_{D}$ is norm continuous at the points of $D^{\prime}$. By Theorem 1.1 this implies that $\phi$ is norm continuous at the points of $D^{\prime}$ and we conclude that $X$ is a GGC space (GC space).

The following three-space property is immediate.

COROLlaRY 3.8. Consider a Banach space $X$ with a closed linear subspace $M$ which is a GGC space (GC space). Then $X$ is a GGC space (GC space) if $X / M$ is a GGC space (GC space).

Again using the fact that $\ell_{\infty}(\Gamma)$ where $\Gamma$ is infinite, is not a GGC space, it follows from Corollary 3.8 that any quotient of $\ell_{\infty}(\Gamma)$ by a GGC space is not a GGC space. In particlar, $\ell_{\infty}(\Gamma) / c_{o}(\Gamma)$ is not a GGC space.

The mapping property associated with that given in Theorem 3.7 holds only for a special case. 
THEOREM 3.9. Consider a continuous linear mapping $T$ from a GGC space (GC space) $X$ onto a Banach space $Y$. If $\operatorname{ker} T$ is reflexive then $Y$ is a GGC space (GC space).

PROOF. By Theorem 2.9 (ii), it is sufficient to consider a minimal weak * usco (minimal weak * cusco) $\Phi$ from a complete metric space $A$ into subsets of $B\left(Y^{* *}\right)$ where the set $\{t \in A: \Phi(t) \cap \widehat{Y} \neq \emptyset\}$ is residual in $A$. Since $T$ maps $X$ onto $Y$ then the second conjugate $T^{\prime \prime}$ maps $X^{* *}$ onto $Y^{* *}$. Since $T^{\prime \prime}$ is an open mapping there exists an $r>0$ such that $T^{\prime \prime}\left(r B\left(X^{* *}\right)\right) \supseteq B\left(Y^{* *}\right)$. We define a mapping $\theta$ from $A$ into subsets of $r B\left(X^{* *}\right)$ by

$$
\theta(t)=\left(T^{\prime \prime}\right)^{-1}(\Phi(t)) \cap r B\left(X^{* *}\right)
$$

Now for each $t \in A, \theta(t)$ is non-empty, weak $*$ compact (and convex). Further, since $\Phi$ is weak $*$ upper semi-continuous and $T^{\prime \prime}$ is weak $*$ to weak $*$ continuous, then $\theta$ has closed graph. But $\theta(A) \subseteq r B\left(X^{* *}\right)$ so $\theta$ is weak * upper semi-continuous. By Zorn's Lemma there exists a minimal weak * usco (minimal weak * cusco) $\theta^{\prime}$ whose graph is contained in that of $\theta$. But further, we see that for each $t \in A$, $T^{\prime \prime} \theta^{\prime}(t) \subseteq T^{\prime \prime} \theta(t) \subseteq \Phi(t)$. Since $\Phi$ is a minimal weak * usco (minimal weak * cusco), then $T^{\prime \prime} \theta^{\prime}=\Phi$.

To show that $\Phi$ is single-valued and norm upper semi-continuous at the points of a residual subset of $A$ we need only show that $\theta^{\prime}$ is single-valued and norm upper semi-continuous at the points of a residual subset of $A$.

Consider $t_{0} \in\{t \in A: \Phi(t) \cap \widehat{Y} \neq \emptyset\}$ and $\hat{y} \in \Phi\left(t_{0}\right) \cap \widehat{Y}$ and $F \in \theta^{\prime}\left(t_{0}\right)$ such that $T^{\prime \prime}(F)=\hat{y}$. Since $T$ is onto there exists $x \in X$ such that $T x=y$ so $F-\hat{x} \in \operatorname{ker} T^{\prime \prime}$. But $\operatorname{ker} T^{\prime \prime}=(\operatorname{ker} T)^{\perp \perp}=w^{*}-c l \widehat{\operatorname{ker} T}$ in $X^{* *}$. Since $\operatorname{ker} T$ is reflexive $w^{*}-c l \widehat{\operatorname{ker} T} T=$ $\widehat{\operatorname{ker} T}$. Then $F \in \hat{x}+\widehat{\operatorname{ker}} T \subseteq \widehat{X}$. So we conclude that $\{t \in A: \Phi(t) \cap \widehat{Y} \neq \emptyset\} \subseteq$ $\{t \in A: \theta(t) \cap \widehat{X} \neq \emptyset\}$ which is therefore residual in $A$. Since $X$ is a GGC space (GC space), then $\theta^{\prime}$ is single-valued and norm upper semi-continuous at the points of a residual subset of $A$ and we conclude that $Y$ is a GGC space (GC space).

So the associated conditional three-space property holds.

COROLlARY 3.10. Consider a GGC space (GC space) $X$ with a closed linear subspace $M$. Then $X / M$ is a $\mathrm{GGC}$ space (GC space) if $M$ is reflexive.

In general the quotient of a GGC space (GC space) is not a GGC space (GC space). In particular, $\ell_{\infty}(\Gamma)$ where $\Gamma$ is infinite, can be represented as the quotient of a GGC space. To show this we need the theory we develop in Section 4. 


\section{Conditions sufficient for membership of the classes of GGC and GC spaces}

In $[8, \mathrm{p} .424]$ a general fragmentability condition was given for a Banach space to be a GC space. Here we give a similar sufficiency condition for both GGC and GC spaces but restrict ourselves to the topology of the unit sphere.

Given a topological space $X$ we say that a function $\lambda: X \times X \rightarrow \mathbb{R}$ is a premetric on $X$ if

(i) $\lambda(x, y) \geq 0$ for all $x, y \in X$, and

(ii) $\lambda(x, y)=0$ if and only if $x=y$.

We define the $\lambda$-topology on $X$ as follows. A subset $U$ of $X$ is said to be $\lambda$-open if for every $x_{0} \in U$ there exists an $r>0$ such that $\left\{x \in X: \lambda\left(x, x_{0}\right)<r\right\} \subseteq U$. Given $x_{0} \in X$ and $\epsilon>0$, a subset of the form $\left\{x \in X: \lambda\left(x, x_{0}\right)<\epsilon\right\}$ is fundamental in defining the $\lambda$-topology but is not necessarily $\lambda$-open. For a non-empty subset $E$ of $X$ we define

$$
\lambda-\operatorname{diam} E \equiv \sup \{\lambda(x, y): x, y \in E\} .
$$

We note that the $\lambda$-topology on a subset $E$ of $X$ is stronger than the relative topology on $E$ if for every $x_{0} \in E$ and open set $W$ containing $x_{0}$ there exists a $\delta>0$ such that $\left\{x \in E: \lambda\left(x, x_{0}\right)<\delta\right\} \subseteq W \cap E$.

Our sufficiency conditions depend on the following general fragmentability properties for topological and linear topological spaces. Given a set $E$ in a linear topological space $X$ and an open half-space $W$ in $X$ where $E \cap W \neq \emptyset$, we call the set $E \cap W$ a slice of $E$.

LEMMA 4.1. (i) Consider a minimal mapping $\phi$ from a Baire space A into subsets of a topological space $X$ and a premetric $\lambda$ on $X$ whose topology on $X$ is stronger than the given topology on $X$. If every non-empty subset of $X$ has relatively open subsets of arbitrarily small $\lambda$-diameter then $\phi$ is single-valued and upper semi-continuous at the points of a dense $G_{\delta}$ subset of $A$.

(ii) Consider a hyperplane minimal mapping $\phi$ from a Baire space A into subsets of $X$ a subset of a linear topological space and a premetric $\lambda$ on $X$ whose topology on $X$ is stronger than the relative linear topology on $X$. If every non-empty subset of $X$ has slices of arbitrarily small $\lambda$-diameter then $\phi$ is single-valued and upper semi-continuous at the points of a dense $G_{\delta}$ subset of $A$.

ProOF. Given $\epsilon>0$, consider the set

$$
\left.O_{\epsilon} \equiv \bigcup \text { \{open sets } V \text { in } A \text { such that } \lambda \text { - } \operatorname{diam} \phi(V)<\epsilon\right\} \text {. }
$$

Now $O_{\epsilon}$ is open; we show that it is dense. Consider any non-empty open set $U$ in $A$. 
(i) Now there exists an open set $W$ in $X$ such that $\lambda$-diam $(W \cap \phi(U))<\epsilon$. But since $\phi$ is minimal there exists a non-empty open set $V \subseteq U$ such that $\phi(V) \subseteq$ $W \cap \phi(U)$ so $\lambda$ - $\operatorname{diam} \phi(V)<\epsilon$.

(ii) Similarly, there exists a slice of $\phi(V)$ with $\lambda$-diameter less than $\epsilon$. Since $\phi$ is hyperplane minimal there exists a non-empty open set $V \subseteq U$ such that $\phi(V)$ lies inside this slice so $\lambda$-diam $\phi(V)<\epsilon$.

In both cases $O_{\epsilon}$ is dense in $A$. Then $\phi$ is single-valued on $\bigcap_{n=1}^{\infty} O_{1 / n}$ and since the $\lambda$-topology is stronger than the given topologies, $\phi$ is upper semi-continuous at the points of $\bigcap_{n=1}^{\infty} O_{1 / n}$.

Our first sufficiency condition follows directly from Lemma 4.1 and Theorem 2.9 (iv).

THEOREM 4.2. A Banach space $X$ is a GGC space (GC space) if there exists an equivalent norm on $X$ and a premetric $\lambda$ on $S(X)$ where the $\lambda$-topology on $S(X)$ is stronger than the weak topology on $S(X)$ and every subset of $S(X)$ has relatively weak open subsets of arbitrarily small $\lambda$-diameter, (slices of arbitrarily small $\lambda$-diameter).

A Banach space $X$ is said to be locally uniformly rotund if given $x_{0} \in S(X)$ and $\epsilon>0$ there exists a $\delta\left(\epsilon, x_{0}\right)>0$ such that $\left\|x_{0}-y\right\|<\epsilon$ when $y \in S(X)$ and $\left\|x_{0}+y\right\|>2-\delta$. This theorem implies that all Banach spaces which can be equivalently renormed to be locally uniformly rotund which includes all separable and weakly compactly generated spaces are GC spaces [8, p. 426]. Furthermore a Banach space $X$ is said to have a Kadec norm if the norm and weak topologies agree on $S(X)$. So this theorem implies that all Banach spaces which can be equivalently renormed to have a Kadec norm are GGC spaces. Interestingly it is known that not all Banach spaces with a Kadec norm can be equivalently renormed to be locally uniformly rotund, [3, p. 325].

We now introduce fragmentability conditions more general than those used in Theorem 4.2. A topological space $X$ is said to be $\sigma$-fragmentable by a premetric $\lambda$ if for each $\epsilon>0$ we can write $X=\bigcup_{k=1}^{\infty} X_{k}$ where for each $k \in \mathbb{N}, X_{k}$ has the property that for each non-empty subset $E_{k}$ of $X_{k}$ there exists a non-empty relatively open subset $U_{k}$ of $E_{k}$ such that $\lambda$-diam $U_{k}<\epsilon$. We say that $X_{k}$ is fragmentable down to $\epsilon$. We say that a subset $X$ of a locally convex space is $\sigma$-slice fragmentable by a premetric $\lambda$ if for each $\epsilon>0$ we can write $X=\bigcup_{k=1}^{\infty} X_{k}$ where for each $k \in \mathbb{N}, X_{k}$ has the property that for each non-empty subset $E_{k}$ of $X_{k}$ there exists a slice of $E_{k}$ of $\lambda$-diameter less than $\epsilon$. We say that $X_{k}$ is slice fragmentable down to $\epsilon$.

We now establish general properties for these $\sigma$-fragmentability conditions similar to those for fragmentability given in Lemma 4.1.

LEMMA 4.3. (i) Given a minimal mapping $\phi$ from a Baire space A into a regular 
Hausdorff space $X$ which is $\sigma$-fragmented by a premetric $\lambda$ whose topology on $X$ is stronger than the given topology on $X$, then $\phi$ is single-valued and upper semicontinuous at the points of a dense $G_{\delta}$ subset of $A$.

(ii) Given a hyperplane minimal mapping $\phi$ from a Baire space $A$ into subsets of $X$ a subset of a separated locally convex space which is $\sigma$-slice fragmentable by $a$ premetric $\lambda$ whose topology on $X$ is stronger than the relative locally convex topology on $X$, then $\phi$ is single-valued and upper semi-continuous at the points of a dense $G_{\delta}$ subset of $A$.

PROOF. By Theorem 1.1, it is sufficient to consider a single-valued minimal (hyperplane minimal) mapping $\phi$ from a Baire space $A$ into $X$. We show that $\phi$ is continuous at the points of a residual subset $R$ of $A$. Given $n \in \mathbb{N}$, consider $X=\bigcup_{k=1}^{\infty} X_{k}$ where for each $k \in \mathbb{N}$,

(i) $X_{k}$ is fragmentable down to $1 / n$, and we define the set $R_{n}$ in $A$ by

$$
R_{n} \equiv\left\{\begin{array}{ll}
t \in A: & \text { there exists an open neighbourhood } V \text { of } t \text { such that } \\
\phi(V) \subseteq \overline{\{x \in X: \lambda(x, \phi(t))<1 / n\}}
\end{array}\right\},
$$

(ii) $X_{k}$ is slice fragmentable down to $1 / n$, and we define the set $R_{n}$ in $A$ by

$$
R_{n} \equiv\left\{\begin{array}{r}
t \in A: \quad \text { there exists an open neighbourhood } V \text { of } t \text { such that } \\
\phi(V) \subseteq \overline{c o}\{x \in X: \lambda(x, \phi(t))<1 / n\}
\end{array}\right\} .
$$

Suppose that $R_{n}$ is not residual in $A$. Then $S \equiv A \backslash R_{n}$ is second category in $A$. Writing $D_{k} \equiv \phi^{-1}\left(X_{k}\right)$ for each $k \in \mathbb{N}$ we have

$$
A=\bigcup_{k=1}^{\infty}\left\{\left(\text { int } \bar{D}_{k} \cap D_{k}\right) \cup\left(D_{k} \backslash \text { int } \bar{D}_{k}\right)\right\} .
$$

So

$$
S=\left(\bigcup_{k=1}^{\infty}\left(D_{k} \cap S\right) \cap \operatorname{int} \bar{D}_{k}\right) \cup\left(\bigcup_{k=1}^{\infty}\left(D_{k} \backslash \operatorname{int} \bar{D}_{k}\right) \cap S\right) .
$$

Now the second term is of the first category so the first term is of the second category. Furthermore, for some $k \in \mathbb{N},\left(D_{k} \cap S\right) \cap$ int $\bar{D}_{k}$ is of second category in $A$.

Consider the set

$$
O_{1 / n} \equiv \bigcup\left\{\text { open sets } V \subseteq \operatorname{int} \bar{D}_{k}: \lambda \text {-diam } \phi\left(V \cap D_{k}\right)<1 / n\right\} .
$$

Now $O_{1 / n}$ is open; we show that it is dense in int $\bar{D}_{k}$. Consider a non-empty open set $U \subseteq$ int $\bar{D}_{k}$. Then $U \cap D_{k} \neq \emptyset$.

(i) There exists a non-empty relatively open subset $U_{k}$ of $\phi\left(U \cap D_{k}\right)$ in $X_{k}$ such that $\lambda$-diam $U_{k}<1 / n$. Since $\phi$ is minimal on $A$ it is minimal on $D_{k}$ which is dense in int $\bar{D}_{k}$, so there exists a non-empty open set $V \subseteq U$ such that $\phi\left(V \cap D_{k}\right) \subseteq U_{k}$ so $\lambda$-diam $\phi\left(V \cap D_{k}\right)<1 / n$. 
(ii) There exists a slice of $\phi\left(U \cap D_{k}\right)$ with $\lambda$-diameter less than $1 / n$. Since $\phi$ is hyperplane minimal on $A$ it is hyperplane minimal on $D_{k}$ which is dense in int $\bar{D}_{k}$, so there exists a non-empty open set $V \subseteq U$ such that $\phi\left(V \cap D_{k}\right)$ lies in the slice so $\lambda$-diam $\phi\left(V \cap D_{k}\right)<1 / n$.

In both cases we conclude that $O_{1 / n}$ is dense in int $\bar{D}_{k}$ and so is residual in int $\bar{D}_{k}$. However, $\left(D_{k} \cap S\right) \cap \operatorname{int} \bar{D}_{k}$ is second category in int $\bar{D}_{k}$, and so we have that $\left(D_{k} \cap S \cap\right.$ int $\left.\bar{D}_{k}\right) \cap O_{1 / n} \neq \emptyset$. This implies that for any $t_{0} \in\left(D_{k} \cap S \cap \operatorname{int} \bar{D}_{k}\right) \cap O_{1 / n}$ there exists an open neighbourhood $V$ of $t_{0}$ such that $V \subseteq$ int $\bar{D}_{k}$ and $\lambda$-diam $\phi\left(V \cap D_{k}\right)<1 / n$. Since $D_{k}$ is dense in $V$ we have, using Theorem 1.1,

(i) when $\phi$ is minimal, $\phi(V) \subseteq\left\{x \in X: \lambda\left(x, \phi\left(t_{0}\right)\right)<1 / n\right\}$

(ii) when $\phi$ is hyperplane minimal, $\phi(V) \subseteq \overline{c o}\left\{x \in X: \lambda\left(x, \phi\left(t_{0}\right)\right)<1 / n\right\}$.

In either case this implies that $t_{0} \in R_{n}$ and so $R_{n} \cap S \neq \emptyset$ which contradicts the definition of $S$. So we conclude that $R_{n}$ is residual in $A$. Then $R \equiv \bigcap_{n=1}^{\infty} R_{n}$ is residual in $A$.

We now show that $\phi$ is continuous at points of $R$.

(i) Consider $t_{0} \in R$ and $W$ an open neighbourhood of $\phi\left(t_{0}\right)$ and since $X$ is regular, an open neighbourhood $U$ of $\phi\left(t_{0}\right)$ such that $U \subseteq \bar{U} \subseteq W$. Since the $\lambda$-topology is stronger on $X$ than the regular topology on $X$ there exists an $n \in \mathbb{N}$ such that $\left\{x \in X: \lambda\left(x, \phi\left(t_{0}\right)\right)<1 / n\right\} \subseteq U$. Now $t_{0} \in R_{n}$ so there exists an open neighbourhood $V$ of $t_{0}$ such that $\phi(V) \subseteq \overline{\left\{x \in X: \lambda\left(x, \phi\left(t_{0}\right)\right)<1 / n\right\}} \subseteq \bar{U} \subseteq W$.

(ii) Consider $t_{0} \in R$ and $W$ an open neighbourhood of $\phi\left(t_{0}\right)$ and since the locally convex space is regular, a convex neighbourhood $U$ of $\phi\left(t_{0}\right)$ such that $U \subseteq$ $\bar{U} \subseteq W$. Since the $\lambda$-topology on $X$ is stronger than the relative locally convex topology on $X$ there exists an $n \in \mathbb{N}$ such that $\left\{x \in X: \lambda\left(x, \phi\left(t_{0}\right)\right)<1 / n\right\} \subseteq U$. Now $t_{0} \in R_{n}$ so there exists an open neighbourhood $V$ of $t_{0}$ such that $\phi(V) \subseteq$ $\overline{\mathrm{co}}\left\{x \in X: \lambda\left(x, \phi\left(t_{0}\right)\right)<1 / n\right\} \subseteq \bar{U} \subseteq W$.

Our second sufficiency condition follows from Lemma 4.3 and Theorem 2.9 (iv).

THEOREM 4.4. A Banach space $X$ is a GGC space (GC space) if there exists an equivalent norm on $X$ and a premetric $\lambda$ on $S(X)$ where the $\lambda$-topology on $S(X)$ is stronger than the weak topology on $S(X)$ and $S(X)$ is $\sigma$-fragmentable by $\lambda,(S(X)$ is $\sigma$-slice fragmentable by $\lambda$ ).

In [11, p. 207] it is shown that if $K$ is any compact Hausdorff space then every odd dual of $\mathscr{C}(K), \mathscr{C}(K)^{(k)}$ with its weak topology and $k$ odd, is $\sigma$-fragmentable by the norm. Theorem 4.4 implies that such spaces are GGC spaces.

In particular, for $\Gamma$ infinite, $\mathscr{C}\left(B\left(\ell_{\infty}(\Gamma)\right) \text {, weak }\right)^{*}$ is a GGC space and we can use this result to show that in general the quotient of a GGC space is not necessarily a GGC space. Consider $T$ the isometric embedding of $\ell_{1}(\Gamma)$ into 
$\left(\mathscr{C}\left(B\left(\ell_{\infty}(\Gamma)\right)\right.\right.$, weak $\left.\left.{ }^{*}\right)\right)$. The conjugate $T^{\prime}$ maps $\mathscr{C}\left(B\left(\ell_{\infty}(\Gamma)\right)\right)^{*}$ onto $\ell_{1}^{*}(\Gamma) \cong$ $\ell_{\infty}(\Gamma)$. So $\mathscr{C}\left(B\left(\ell_{\infty}(\Gamma)\right)\right)^{*} / \operatorname{ker} T^{\prime} \cong \ell_{\infty}(\Gamma)$.

Using the fact that $\ell_{\infty}(\Gamma)$, where $\Gamma$ is infinite, is not a GGC space, it follows from Theorem 4.4 that $\ell_{\infty}(\Gamma)$ is not $\sigma$-fragmentable. We showed after Corollary 3.4 that $\ell_{\infty}(\Gamma) / c_{0}(\Gamma)$ is not a GGC space. Again from Theorem 4.4 we can deduce that this space is not $\sigma$-fragmentable.

\section{Spaces which are not GGC spaces or GC spaces}

In their paper [10, p. 201] the authors showed that a Banach space which possesses a family of subsets satisfying particular properties, cannot be $\sigma$-fragmented by the norm. Using such an idea, Namioka has been able to show that such spaces are not GGC spaces. In the paper [12, p. 214] they showed that $\ell_{\infty}(\mathbb{N})$ possesses such a family of subsets and so Namioka has deduced that $\ell_{\infty}(\mathbb{N})$ is not a GGC space. In paper [8, Section 3] it was shown that $\ell_{\infty}(\Gamma)$ where $\Gamma$ is uncountable, is not a GC space and to do this a counter-example was constructed using the Banach-Mazur game. Here we exploit the similarity of both techniques to give a characterisation of non-membership of our generic continuity classes and derive Namioka's result.

Given a Banach space $X$ and a family $\mathscr{G}$ of non-empty open subsets of $B(X)$, we denote by $\mathscr{S}$ a non-empty family of non-empty subsets of $B(X)$ which satisfy properties:

(i) if $S \in \mathscr{S}$ and $W \in \mathscr{G}$ such that $S \cap W \neq \emptyset$ then there exists a $T \in \mathscr{S}$ such that $T \subseteq S \cap W$,

(ii) $\inf \{\operatorname{diam} S: S \in \mathscr{S}\}>0$,

(iii) $\bigcup\{S: S \in \mathscr{S}\} \in \mathscr{S}$.

We now define what we will call an $\mathscr{S}-\mathscr{G}$ game on $B(X)$. This is a game between two players $\alpha$ and $\beta$. Player $\alpha$ begins by choosing $S_{1}=\bigcup\{S: S \in \mathscr{S}\}$ and player $\beta$ responds by choosing $V_{1} \in \mathscr{G}$ such that $V_{1} \cap S_{1} \neq \emptyset$. Then $\alpha$ chooses $S_{2} \in \mathscr{S}$ such that $S_{2} \subseteq V_{1} \cap S_{1} \subseteq S_{1}$ and $\beta$ responds by choosing $V_{2} \in \mathscr{G}$ such that $V_{2} \cap S_{2} \neq \emptyset$. Continuing in this way, the sequence of sets

$$
S_{1} \supseteq V_{1} \cap S_{1} \supseteq S_{2} \supseteq V_{2} \cap S_{2} \supseteq \cdots S_{n} \supseteq V_{n} \cap S_{n} \supseteq \cdots
$$

is called a play and is denoted by $p \equiv\left(S_{i}, V_{i}\right)_{i=1}^{\infty}$. We say that $\alpha$ wins this play if $\bigcap_{i=1}^{\infty} S_{i} \neq \emptyset$. A strategy $s$ for the player $\alpha$ is a mapping (or rule) by which $\alpha$ assigns to every partial play

$$
\left(S_{i}, V_{i}\right)_{i=1}^{n} \equiv S_{1} \supseteq V_{1} \cap S_{1} \supseteq S_{2} \supseteq S_{2} \supseteq V_{2} \cap S_{2} \supseteq \cdots \supseteq S_{n} \supseteq V_{n} \cap S_{n}
$$

an element $S_{n+1} \in \mathscr{S}$ such that $S_{n+1} \subseteq V_{n} \cap S_{n}$; that is, $S_{n+1}=s\left(\left(S_{i}, V_{i}\right)_{i=1}^{n}\right)$. We say that the play $p \equiv\left(S_{i}, V_{i}\right)_{i=1}^{\infty}$ is an $s$-play if $\alpha$ plays according to strategy $s$; that is, for 
each $n \in \mathbb{N}, S_{n+1}=s\left(\left(S_{i}, V_{i}\right)_{i=1}^{n}\right)$. The strategy $s$ is said to be an $\alpha$-winning strategy if every $s$-play is won by $\alpha$.

Given an $\alpha$-winning strategy $s$ for the $\mathscr{S}-\mathscr{G}$ game, we can define a metric $d$ on the set $P$ of all $s$-plays, similar to that in [8, Lemma 3.2]. For any $p \equiv\left(S_{i}, V_{i}\right)_{i=1}^{\infty} \in P$ we define $d(p, p)=0$ for each $p \in P$ and if $p^{\prime} \equiv\left(S_{i}^{\prime}, V_{i}^{\prime}\right) \neq\left(S_{i}^{\prime \prime}, V_{i}^{\prime \prime}\right) \equiv p^{\prime \prime}$ then $d\left(p^{\prime}, p^{\prime \prime}\right)=1 / n$ where $n$ is the first natural number $i$ where $S_{i}^{\prime} \neq S_{i}^{\prime \prime}$ or $V_{i}^{\prime} \neq V_{i}^{\prime \prime}$.

LEMMA 5.1. The metric space $(P, d)$ is complete.

Proof. Consider the Cauchy sequence $\left\{p^{n} \equiv\left(S_{i}^{n}, V_{i}^{n}\right)_{i=1}^{\infty}\right\}$ in $P$. Then for every $i \in \mathbb{N}$ there exists some $n_{i} \geq i$ such that the sequence $\left\{n_{i}\right\}$ is increasing and such that $S_{k}^{n_{i}}=S_{k}^{n}$ and $V_{k}^{n_{i}}=V_{k}^{n}$ for all $k \in\{1,2, \ldots, i\}$ and $n \geq n_{i}$. So we define a new play $p^{*}$ by

$$
p^{*} \equiv\left(S_{i}^{*}, V_{i}^{*}\right)_{i=1}^{\infty} \quad \text { where } \quad S_{i}^{*}=S_{i}^{n_{i}} \text { and } V_{i}^{*}=V_{i}^{n_{i}} .
$$

Now for each $i \in \mathbb{N}, S_{i}^{*}=S_{i}^{n_{i}}=s\left(\left(S_{k}^{n_{i}}, V_{k}^{n_{i}}\right)_{k=1}^{i-1}\right)=s\left(\left(S_{k}^{*}, V_{k}^{*}\right)_{k=1}^{i-1}\right)$ so $p^{*}$ is an $s$-play, and we conclude that $d\left(p^{n}, p^{*}\right) \rightarrow \infty$ as $n \rightarrow \infty$.

Given a Banach space $X$, the particular family $\mathscr{G}$ of non-empty open subsets of $B(X)$ which relate to the GGC property is the family $\mathscr{W}$ of non-empty weak open subsets of $B(X)$. We will call a game associated with the family $\mathscr{W}$ an $\mathscr{S}-\mathscr{W}$ game. The particular family $\mathscr{G}$ which relate to the GC property is the family $\mathscr{H}$ of non-empty open half-space subsets of $B(X)$. We will call a game associated with the family $\mathscr{H}$ an $\mathscr{S}-\mathscr{H}$ game.

We are now ready to give our characterisation theorem in terms of $\mathscr{S}-\mathscr{G}$ games.

THEOREM 5.2. A Banach space $X$ is not a GGC space (GC space) if and only if there exists an $\mathscr{S}-\mathscr{W}$ game $(\mathscr{S}-\mathscr{H}$ game) where there is an $\alpha$-winning strategy.

Proof. Suppose that $X$ is not a GGC space (GC space). Then there exists a weakly minimal (hyperplane minimal) mapping $\phi$ from a complete metric space $A$ into nonempty subsets of $B(X)$ which is not single-valued and norm upper semi-continuous on any dense $G_{\delta}$ subset of $A$. It follows that for some $\epsilon>0$, the set

$$
\left.O_{\epsilon} \equiv \bigcup \text { \{open sets } U \text { in } A \text { such that } \operatorname{diam} \phi(U) \leq \epsilon\right\}
$$

is not dense in $A$, and so for some non-empty open set $W \subseteq A, O_{\epsilon} \cap W=\emptyset$.

We define the family

$$
\mathscr{S} \equiv\{\phi(U): U \text { is a non-empty open subset of } W\} .
$$


Since $\phi(W) \in \mathscr{S}$ then $\mathscr{S}$ is not empty and condition (iii) is satisfied. Furthermore, condition (i) is satisfied for the $\mathscr{W}$ family by the weak minimality of $\phi$ (and for the $\mathscr{H}$ family by the hyperplane minimality of $\phi$ ). Clearly condition (ii) is satisfied since $\operatorname{diam} \phi(U)>\epsilon$ for all non-empty open subsets $U$ of $W$. We need to show that there is an $\alpha$-winning strategy for the $\mathscr{S}-\mathscr{W}$ game ( $\mathscr{S}-\mathscr{H}$ game). Player $\alpha$ begins by choosing $S_{1} \equiv \phi(W) \in \mathscr{S}$. Player $\beta$ responds by choosing a non-empty $V_{1} \in \mathscr{W}\left(V_{1} \in \mathscr{H}\right)$ where $V_{1} \cap S_{1} \subseteq S_{1}$. Then $\alpha$ chooses a non-empty open set $U_{2} \subseteq \bar{U}_{2} \subseteq W$ with $\operatorname{diam} U_{2}<1 / 2^{2}$ and $\phi\left(U_{2}\right) \subseteq V_{1} \cap S_{1}$, this being possible because of the minimality property of $\phi$, and setting $S_{2} \equiv \phi\left(U_{2}\right) \in \mathscr{S}$.

At the beginning of the $n$th stage of this process we have a sequence of non-empty open sets with their closures in $A$,

$$
W \supseteq \bar{U}_{2} \supseteq U_{2} \supseteq \cdots \supseteq \bar{U}_{n-1} \supseteq U_{n-1}
$$

and a sequence of non-empty sets from the $\mathscr{W}$ family ( $\mathscr{H}$ family) and sets from $\mathscr{S}$,

$$
S_{1} \supseteq V_{1} \cap S_{1} \supseteq S_{2} \supseteq V_{2} \cap S_{2} \supseteq \cdots \supseteq S_{n-1} \supseteq V_{n-1} \cap S_{n-1}
$$

such that for each $i \in\{2,3, \ldots, n-1\}$, diam $U_{i}<1 / 2^{i}$ and $\phi\left(U_{i}\right) \subseteq V_{i-1} \cap S_{i-1}$ with $S_{i} \equiv \phi\left(U_{i}\right) \in \mathscr{S}$. Player $\alpha$ then chooses a non-empty open set $U_{n} \subseteq \bar{U}_{n} \subseteq U_{n-1}$ with diam $U_{n}<1 / 2^{n}$ and $\phi\left(U_{n}\right) \subseteq V_{n-1} \cap S_{n-1}$, this being possible because of the minimality property of $\phi$, and setting $S_{n} \equiv \phi\left(U_{n}\right) \in \mathscr{S}$. Now $\bigcap_{i=2}^{\infty} U_{i}=\bigcap_{i=2}^{\infty} \bar{U}_{i} \neq \emptyset$ by Cantor's Intersection Theorem, so $\bigcap_{i=1}^{\infty} S_{i} \supseteq \bigcap_{i=2}^{\infty} \phi\left(U_{i}\right) \supseteq \phi\left(\bigcap_{i=2}^{\infty} U_{i}\right) \neq \emptyset$. We conclude that this is an $\alpha$-winning strategy.

Conversely, suppose that there exists an $\mathscr{S}-\mathscr{W}$ game $(\mathscr{S}-\mathscr{H}$ game) on $B(X)$ with an $\alpha$-winning strategy $s$. We consider $(P, d)$ the complete metric space from Lemma 5.1 and the mapping $\phi$ from $P$ into subsets of $B(X)$ defined for $p \equiv\left(S_{i}, V_{i}\right)_{i=1}^{\infty}$ by $\phi(p)=\bigcap_{i=1}^{\infty} S_{i}$. For each $p \in P, \phi(p) \neq \emptyset$ since $p$ is an $s$-play.

To show that at $\phi$ is weakly minimal (hyperplane minimal) consider a non-empty open set $U$ in $P$ and $W \in \mathscr{W}(W \in \mathscr{H})$ such that $\phi(U) \cap W \neq \emptyset$. For $p \equiv$ $\left(S_{i}, V_{i}\right)_{i=1}^{\infty} \in U$ where $\phi(p) \cap W \neq \emptyset$ we have $S_{i} \cap W \neq \emptyset$ for all $i \in \mathbb{N}$. But also there exists an $n \in \mathbb{N}$ such that $B(p ; 1 / n) \subseteq U$. Consider $p_{1}$ a continuation of the partial $s$-play where $S_{n+1}^{1}=S_{n+1}=s\left(\left(S_{i}, V_{i}\right)_{i=1}^{n}\right)$ and $V_{n+1}^{1}=W$. Then $V \equiv B\left(p_{1} ; 1 /(n+1)\right) \subseteq B(p ; 1 / n) \subseteq U$ and $\phi(V) \subseteq W$.

Suppose that $\inf \{\operatorname{diam} S: S \in \mathscr{S}\}=r>0$. To show that $\phi$ is nowhere singlevalued and norm upper semi-continuous it is sufficient to show that each $p \in P$ and $n \in \mathbb{N}$, diam $\phi(B(p ; 1 / n))>r$. Consider $p \equiv\left(S_{i}, V_{i}\right)_{i=1}^{\infty}$ and $n \in \mathbb{N}$. By the definition of $r, \operatorname{diam} S_{n+1}>r$, so there exists an $f \in X^{*},\|f\|=1$ and $x, y \in S_{n+1}$ such that $|f(x-y)|>r$. Therefore there exist open half-spaces $W_{1}$ and $W_{2}$ defined by $f$ such that $x \in W_{1}$ and $y \in W_{2}$ with the property that $d\left(S_{n+1} \cap W_{1}, S_{n+1} \cap W_{2}\right)>r$. Consider $p_{1}$ a continuation of the partial $s$-play where $S_{n+1}^{1}=S_{n+1}=s\left(\left(S_{i}, V_{i}\right)_{i=1}^{n}\right)$ 
and $V_{n+1}^{1}=W_{1}$ and $p_{2}$ a continuation of the partial $s$-play where $S_{n+1}^{2}=S_{n+1}=$ $s\left(\left(S_{i}, V_{i}\right)_{i=1}^{n}\right)$ and $V_{n+1}^{2}=W_{2}$. Now $p_{1}, p_{2} \in B(p ; 1 / n)$. Therefore,

$$
\begin{aligned}
\operatorname{diam} \phi(B(p ; 1 / n) & \geq \sup \left\{\|x-y\|: x \in \phi\left(p_{1}\right), y \in \phi\left(p_{2}\right)\right\} \\
& \geq d\left(S_{n+1} \cap W_{1}, S_{n+1} \cap W_{2}\right)>r .
\end{aligned}
$$

We conclude that $X$ is not a GGC space (GC space).

As an application of this characterisation theorem we establish Namioka's result.

\section{COROLLARY 5.3. The Banach space $\ell_{\infty}(\mathbb{N})$ is not a GGC space.}

PROOF. Following Theorem 5.2 we need to show that there exists a family $\mathscr{S}$ of subsets of $B\left(\ell_{\infty}\right)$ and an $\mathscr{S}-\mathscr{W}$ game where there is an $\alpha$-winning strategy. Given $x \equiv x(n) \in B\left(\ell_{\infty}\right)$ and set $M \subseteq \mathbb{N}$ with $\mathbb{N} \backslash M$ infinite consider the subset $S$ of $B\left(\ell_{\infty}\right)$ defined for $y \equiv y(n)$ by

$$
S(x, M) \equiv\left\{y \in B\left(\ell_{\infty}\right): x(m)=y(m) \text { for all } m \in M\right\}
$$

and the family

$$
\mathscr{S} \equiv\left\{S(x, M): x \in B\left(\ell_{\infty}\right) \text { and } M \subseteq \mathbb{N} \text { with } \mathbb{N} \backslash M \text { infinite }\right\}
$$

It is proved in $[12$, p. 214] that the family satisfies property (i) of an $\mathscr{S}-\mathscr{W}$ family. Since $\mathbb{N} \backslash M \neq \emptyset$ and $S(x, M)$ has more than one element, diam $S(x, M) \geq$ 2 for every $S(x, M) \in \mathscr{S}$, so property (ii) is clearly satisfied. Furthermore, for any $x \in B\left(\ell_{\infty}\right), S(x, \emptyset)=B\left(\ell_{\infty}\right)=\bigcup\{S: S \in \mathscr{S}\}$ so property (iii) is satisfied. Playing an $\mathscr{S}-\mathscr{W}$ game with the family $\mathscr{S}$ we need to show that there exists an $\alpha$-winning strategy. Consider any decreasing sequence $\left\{S\left(x_{i}, M_{i}\right)\right\}$ in $\mathscr{S}$; that is, $S\left(x_{i}, M_{i}\right) \supseteq S\left(x_{i+1}, M_{i+1}\right)$ for all $i \in \mathbb{N}$. This means that $M_{i} \subseteq M_{i+1}$ and $\left.x_{i}\right|_{M_{i}}=\left.x_{i+1}\right|_{M_{i}}$ for each $i \in \mathbb{N}$. Consider $x$ a common extension of $\left.x_{i}\right|_{M_{i}}$ for each $i \in \mathbb{N}$ to $\bigcup_{i=1}^{\infty} M_{i}$ and zero elsewhere. Then $x \in \bigcap_{i=1}^{\infty} S\left(x_{i}, M_{i}\right)$ and so $\bigcap_{i=1}^{\infty} S\left(x_{i}, M_{i}\right) \neq \emptyset$. So if $\alpha$ follows any strategy $s$ then it will be an $\alpha$-winning strategy. We conclude that $\ell_{\infty}(\mathbb{N})$ is not a GGC space.

In [18, p. 19], it is shown, assuming the continuum hypothesis, that if $K$ is an infinite compact Hausdorff $F$-space then $\mathscr{C}(K)$ contains a subspace topologically isomorphic to $\ell_{\infty}(\mathbb{N})$. In [11, p. 197] it is shown unconditionally that such a $\mathscr{C}(K)$ is not $\sigma$-fragmented by the norm. The following corollary strengthens this result using Theorem 3.5.

COROLLARY 5.4. If $K$ is an infinite compact Hausdorff $F$-space then $\mathscr{C}(K)$ is not a GGC space. 
In the definition of a GGC space (GC space) we require our minimal (hyperplane minimal) mappings to have a complete metric space domain. However, we should note that in the characterisation Theorem 5.2 we only used the fact that the domain space is $\alpha$-favourable; that is, there exists an $\alpha$-winning tactic for the game. So a more general form of this theorem is available.

\section{Problems arising}

There are several significant problems which remain from this investigation.

(1) Can every GC space be equivalently renormed to be locally uniformly rotund?

Of course, a positive solution to this problem would solve several outstanding problems. It would imply that every Banach space with the Radon-Nikodým property, all Banach spaces which can be equivalently renormed to be weakly locally uniformly rotund, or whose dual can be equivalently renormed to be smooth or whose dual belongs to Stegall's class $\mathscr{S}$, could be equivalently renormed to be locally uniformly rotund. So there is every reason to believe that if there is a positive solution to the problem it will be difficult to achieve. In papers [13], [5, 6, 7] and [14], sufficiency conditions for a Banach space to be a GC space were given in terms of a variety of denting point properties of the unit ball. However, it has been shown that two of these properties, that every point of the unit sphere is a denting point ( $\alpha$-denting point) of the unit ball, imply that the space can be equivalently renormed to be locally uniformly rotund, [20, p. 306], [21, p. 179].

(2) Can every GGC space be equivalently renormed to to have a Kadec norm?

Although the papers $[9,10,11]$ give conditions sufficient for a Banach space with its weak topology to be $\sigma$-fragmentable by its norm, including that there exist an equivalent Kadec norm, yet no example has been given which has been shown to possess no equivalent Kadec norm.

(3) Is the class of GGC spaces identical to the class of GC spaces?

Theorem 3.3 defined a class of Banach spaces on which the classes are the same. We should note again that there is an example of a Banach space with a Kadec norm which cannot be equivalently renormed to be locally uniformly rotund, [3, p. 325]. So if there is a positive solution to Problem 1 then there is a negative solution to Problem 3 and if there is a positive solution to Problem 3 then there is a negative solution to Problem 1.

In [13], a class of differentiability spaces was defined. A Banach space $X$ is a dual differentiability space (DD space) if every continuous convex function $\phi$ on an open convex subset $A$ of $X^{*}$ possessing a weak * continuous subgradient at the points of a residual subset of $A$, is Fréchet differentiable on a dense $G_{\delta}$ subset of $A$. The class of DD spaces contains the class of GC spaces. 
(4) Is the class of DD spaces identical to the class of GC spaces?

So far no example has been given of a Banach space which is not a DD space. Professor Namioka's result that $\ell_{\infty}(\mathbb{N})$ is not a GGC space suggests that we determine whether $\ell_{\infty}(\mathbb{N})$ is a DD space. However, the construction of a convex function of the special type which does not satisfy the required properties would be much more difficult than the construction of minimal mappings given so far. Furthermore, the problem is somewhat the same as the outstanding problem of the relation between the class of weak Asplund spaces and those from Stegall's class $\mathscr{S}$.

\section{References}

[1] J. Borwein, S. Fitzpatrick and P. Kenderov, 'Minimal convex uscos and monotone operators on small sets', Canad. J. Math. 43 (1991), 461-476.

[2] J. B. Collier, 'The dual of a space with the Radon-Nikodým property', Pacific J. Math. 64 (1976), 103-106.

[3] R. Deville, G. Godefroy and V. Zizler, Smoothness and renormings in Banach spaces, Pitman Monographs Surveys Pure Appl. Math. 64 (Longmans, Harlow, 1993).

[4] J. R. Giles and M. O. Bartlett, 'Modified continuity and a generalisation of Michael's Selection Theorem', Set-Val. Anal. 1 (1993), 365-378.

[5] J. R. Giles and W. B. Moors, 'A continuity property related to Kuratowski's index of noncompactness, its relevance to the drop property and its implications for differentiability theory', $J$. Math. Anal. Appl. 178 (1993), 247-268.

[6] _ - 'The implications for differentiability of a weak index of non-compactness', Bull. Austral. Math. Soc. 48 (1993), 75-91.

[7] _ - 'Differentiability properties of Banach spaces where the boundary of the closed unit ball has denting point properties ', Proc. Centre Math. Anal. Austral. Nat. Univ. 29 (1992), 107-115.

[8] J. R. Giles, P. S. Kenderov, W. B. Moors and S. D. Sciffer, 'Generic differentiability of convex functions on the dual of a Banach space', Pacific J. Math. 172 (1996), 413-431.

[9] J. E. Jayne, I. Namioka and C. A. Rogers, 'Topological properties of Banach spaces', Proc. London Math. Soc. 66 (1993), 651-672.

[10] —_ ' $\sigma$-fragmentable Banach spaces', Mathematika 39 (1992), 161-188.

[11] _ ' $\sigma$-fragmentable Banach spaces', Mathematika 39 (1992), 197-215.

[12] - 'Fragmentability and $\sigma$-fragmentabilty', Fund. Math. 143 (1993), 207-220.

[13] P. S. Kenderov and J. R. Giles, 'On the structure of Banach spaces with Mazur's intersection property', Math. Ann. 291 (1991), 463-471.

[14] W. B. Moors, 'A continuity property related to an index of non-separability and its applications', Bull. Austral. Math. Soc. 46 (1992), 67-79.

[15] I. Namioka, 'Separate continuity and joint continuity', Pacific J. Math. 51 (1974), 515-531.

[16] R. R. Phelps, Convex functions, monotone operators and differentiability, Lecture Notes in Math. 1364, 2nd edition, (Springer, Berlin, 1993).

[17] David Preiss, R. R. Phelps and I. Namioka, 'Smooth Banach spaces, weak Asplund spaces and monotone or usco mappings', Israel J. Math. 72 (1990), 257-279.

[18] H. Rosenthall, 'On relatively disjoint families of measures, with some applications to Banach space theory', Studia Math. 37 (1970), 13-36. 
[19] M. A. Smith, 'A curious generalisation of local uniform rotundity', Comment. Math. Univ. Carolin. 25 (1984), 659-665.

[20] S. Troyanski, 'On a property of the norm which is close to local uniform rotundity', Math. Ann. 271 (1985), 305-314.

[21] _ _ ' 'On some generalisations of denting points', Israel J. Math. 88 (1994), 175-188.

Department of Mathematics

The University of Auckland

New Zealand
Department of Mathematics

The University of Newcastle

NSW 2308

Australia 